\title{
Differential Mobile Robot Controller Study: A Low Cost Experiment Based on a Small Arduino Based Prototype
}

\author{
José Gonçalves ${ }^{1}$ and Paulo Costa ${ }^{2}$
}

\begin{abstract}
In this paper it is presented a low cost experiment based on a small Arduino based prototype. The chosen educational challenge is a classical introductory experiment, that consists in following a line with a mobile robot. The presented experiment has as goal to introduce students to mobile robotics, having as base a challenge and a kinematics that are commonly applied in Junior competitions. A group of students participated in a workshop that consisted, initially, in a lecture where tutors explained the differential robot kinematics and how to develop a controller for the proposed challenge. Then the students, after the theoretical introduction, implemented the proposed robot controller.
\end{abstract}

\section{INTRODUCTION}

In this paper it is presented an educational mobile robotics experiment based on a low cost mobile robot prototype apply, being shown in Figure 1. The mobile robot, consists in a 3D printed small prototype, that uses inexpensive hardware, such as servo motors, an Arduino Uno platform and an infra-red detector array. For the proposed robot, continuous rotation is necessary, so the servo motors must be modified. This modification consists in disconnecting the position potentiometer from the gear train, setting the potentiometer to a fixed position, and removing the angle stops from the motor shaft. The robot is equipped with the Zumo reflectance sensor, which provides an easy way to add line sensing or edge detection. The prototyped robot has the possibility to communicate with external devices, allowing remote control and real-time monitoring. An external vision based global localization system was also developed, in order to provide, in real-time, the robot absolute localization, allowing the estimate of some robot parameters.

The external Localization system provides the real time robot localization, that can be sent by UDP packets over the Internet. The computer application that is applied to change the controller and robot parameters, has access to the robot localization Data, as shown in Figure 2, being a valuable information in order to estimate some robot parameters. The computer application has the ability to monitor the robot sensors, facilitating the robot Software Debug. The wireless link is provided by an nRF24L01 based module, connected to the SPI Port.

The chosen educational challenge is a classical introductory mobile robotics experiment, that consists in following a line with a mobile robot based on the differential kinematics

Both authors are Senior Researchers at CROB from INESC-TEC

${ }^{1}$ José Gonçalves is with the Polytechnic Institute of Bragança, Portugal goncalves@ipb.pt

${ }^{2}$ Paulo Costa is with the Faculty of Engineering of the University of Porto pacolfe.up.pt

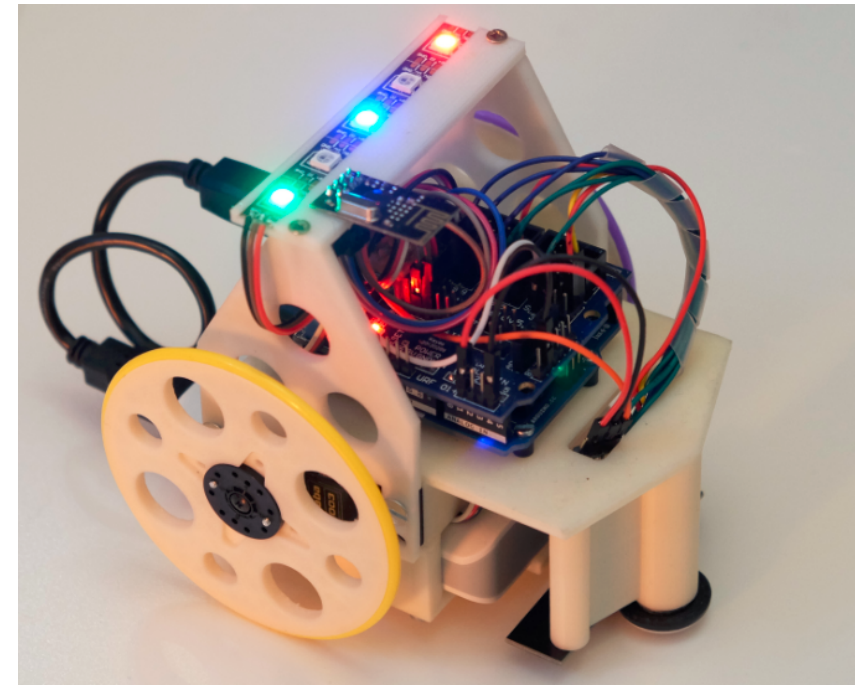

Fig. 1. Robot Prototype.

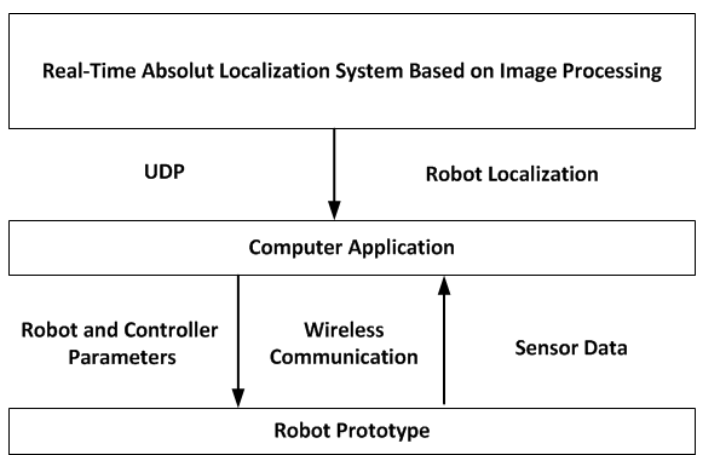

Fig. 2. System Arquitecture

[1] [2] [3] [4], which is commonly used in Junior competitions [5] [6] [7] [8]. A group of students participated in a workshop that consisted initially in a lecture where tutors explained the differential kinematics and how to develop a robot controller for the proposed challenge. Then the students, after the theoretical introduction, implemented a controller for a provided robot prototype.

The paper is structured as follows, after a brief introduction the robot prototype and the external global vision system are described, then the educational experiment is presented and finally some conclusions and future work are presented.

\section{Robot PROTOTYPE DESCRIPTION}

The prototyped mobile robot consists in a $3 \mathrm{D}$ printed small prototype, that uses inexpensive hardware, such as servo 
motors, an Arduino Uno platform and an infra-red detector array, the robot prototype components are shown in Figure 3.

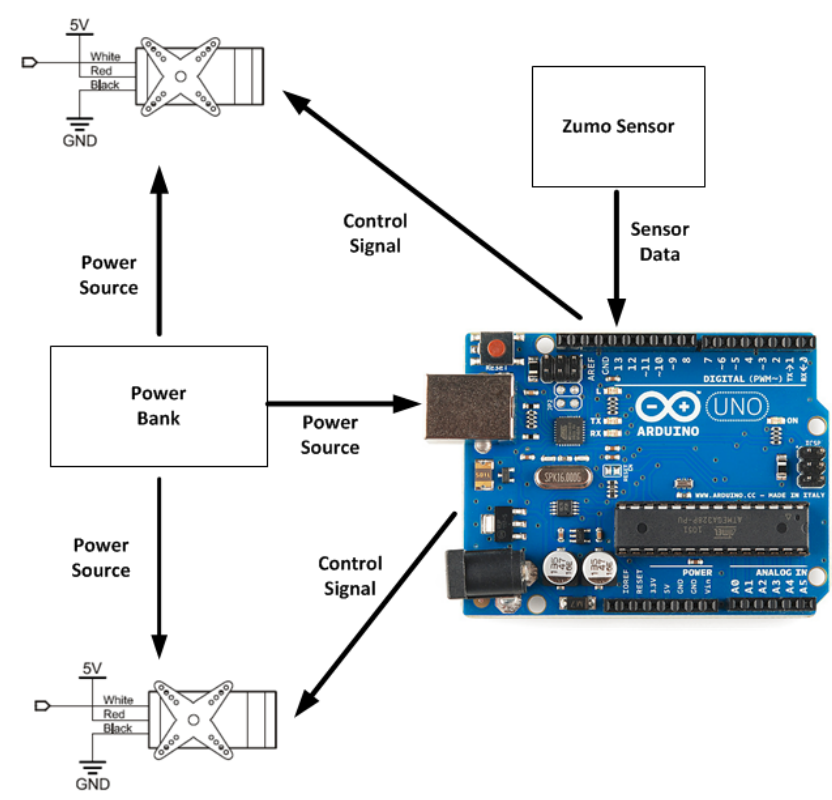

Fig. 3. Robot Prototype Components

The 3D printer models, that were developed in order to prototype the robot, are presented in Figure 4, where it can be seen the $3 \mathrm{D}$ models for the robot chassis and wheels $3 \mathrm{D}$ models.

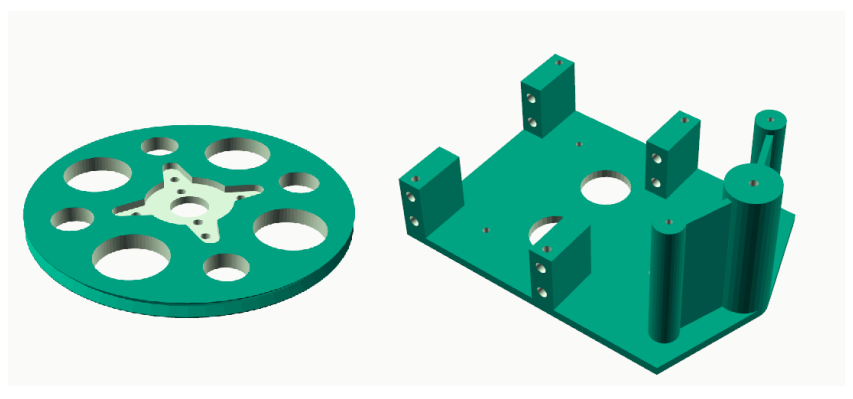

Fig. 4. Robot Prototype 3D Printer Models.

In the next subsections it will be introduced the prototype kinematics and its sensors and actuators description.

\section{A. Prototype Kinematics}

The differential robot, presented in Figure 5, is composed by two wheels, with their shaft passing by the same axle. Their movement is controlled by varying each wheel velocity independently.

The mechanical structure of the differential robot, presented in Figure 5, does not allow movements along the axle that cross wheel shafts. Considering that there is no lateral slip, each wheel velocity in the wheels ground contact point is perpendicular to the axle along the wheel shaft.

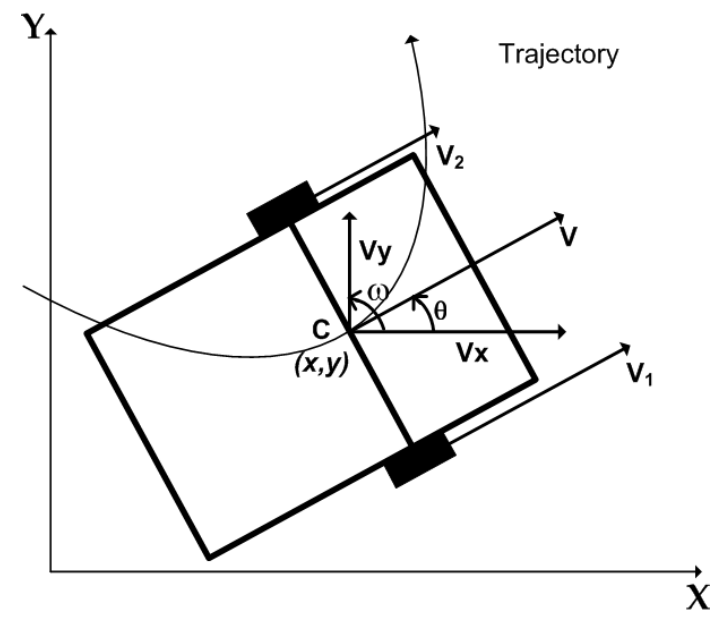

Fig. 5. Differential Robot

In this case $V_{1}(t)$ and $V_{2}(t)$ are the velocities measured in the contact point between the wheel and the ground. The robot's linear and angular velocities are given by equations 1 and 2.

$$
\begin{aligned}
& v(t)=\frac{V_{1}(t)+V_{2}(t)}{2} \\
& \omega(t)=\frac{V_{1}(t)-V_{2}(t)}{b}
\end{aligned}
$$

where $b$ represents the distance between the ground contact points.

Considering the non slip situation, for a global localization reference system, the differential robot kinematics can be described by the following equations.

$$
\begin{gathered}
\dot{x}=v(t) \cos (\theta(t)) \\
\dot{y}=v(t) \sin (\theta(t)) \\
\dot{\theta}=\omega(t)
\end{gathered}
$$

\section{B. Sensors and Actuators}

1) Sensor: The robot is equipped with the Zumo reflectance sensor, shown in Figure 6, providing an easy way to add line sensing or edge detection. It features six separate reflectance sensors, each consisting of an IR emitter coupled with a phototransistor that responds based on how much emitter light is reflected back to it. The purpose of using the referred sensor is to sense and follow a line.

The procedure for reading each sensor is as follows:

- Make the I/O line connected to that sensor an output and drive it high.

- Wait several microseconds to give capacitor node time to reach $5 \mathrm{~V}$.

- Make the I/O line an input (with internal pull-up disabled). 


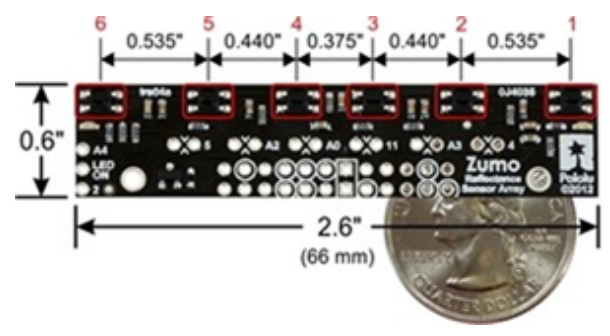

Fig. 6. Zumo reflectance sensor [9]

- Measure the time for the voltage to decay by waiting for the I/O line to go low.

With a strong reflectance, the decay time can be as low as several dozen microseconds; with no reflectance, the decay time can be up to a few milliseconds. More information about this sensor can be found in [9].

2) Actuator: The robot actuator is the Futaba $\mathrm{S} 3003$ Servo. A servo motor is a complete assembly made of a small high RPM motor, gear reduction, H-Bridge and position control circuitry. If the servo is not modified it is used to produce a rotational position based on a Pulse Width Modulated (PWM) signal. The Futaba S3003 servo motor has three inputs: PWM (white), power (red), and ground (black). Based on the PWM signal the servo will turn its shaft to a position within a range of approximately 200 degrees. When a PWM command is given to the circuitry an error signal is produced. This error signal turns the motor in the appropriate direction. The motor gearing turns a position potentiometer, which gives a feedback signal to the position control circuitry. When the correct position is indicated by the potentiometer, the error signal becomes small enough, so the motor stops turning. For the proposed robot, continuous rotation is necessary, so the servo motors must be modified. This modification consists in disconnecting the position potentiometer from the gear train, setting the potentiometer for a known PWM signal and removing the angle stops from the motor shaft. Some offset developed by software is necessary to get the two motors to turn at the same speed. More detailed information of the Futaba S3003 servo motor and its modification can be found in [10].

In order to obtain the actuator model it was necessary to know for each control signal the output velocity of each modified servo-motor, incremental encoders were used for that purpose. The use of incremental encoders, as shown in Figure 7, was only necessary to obtain the actuator model. The used incremental encoders are an expensive piece of hardware that would increase considerably the cost of the robot prototype. A tachometer was used in order to convert the measured transitions per sample time to Rad/s. In order to measure the motor angular velocity with the tachometer, a printed black and white pattern with transitions was attached to a robot wheel.

The control signal is the same as for a standard servo, only this time the length of the on time pulse will affect the speed and directions. For a certain pulse width the servo will stop. Values above or below will make the servo rotate faster in

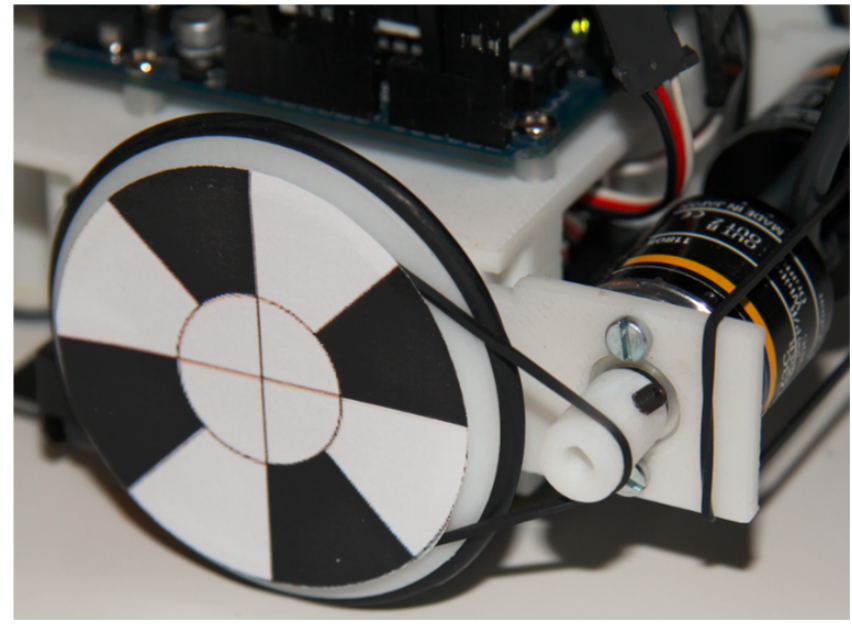

Fig. 7. Robot with encoders.

either direction. The signal $(d)$, depicted in Figure 8, is the difference for the stopping pulse width. This value must be divided by 40000 , in order to obtain the time in seconds. As there is a gearbox with an high ratio, the dynamic response is very fast. The most important aspect of the model is the non linearity introduced by the modified controller. This non linearity can be seen in Figure 8 where the steady state speed for a certain pulse width has a small dead zone and a non linear behavior as it approaches the maximum speed. In order to model these non linearities, equation 6 , saturated for values inferior to zero, was estimated. Using experimental speed measures the best fit was found by optimizing the values of $a_{2} . . a_{0}, b_{2} . . b_{0}$. The total error, being the sum of the absolute differences, was used as the target function. The estimated values can be seen in Table I [11].

$$
\omega(d)=\frac{a_{2} \cdot d^{2}+a_{1} \cdot d+a_{0}}{b_{2} \cdot d^{2}+b_{1} \cdot d+b_{0}}
$$

\begin{tabular}{|c|c|}
\hline Parameters & Value \\
\hline$a_{1}$ & $-34.760 \mathrm{E}-6$ \\
\hline$a_{2}$ & $-69.581 \mathrm{E}-3$ \\
\hline$a_{3}$ & $488.777 \mathrm{E}-3$ \\
\hline$b_{1}$ & $-29.663 \mathrm{E}-6$ \\
\hline$b_{2}$ & $2.278 \mathrm{E}-3$ \\
\hline$b_{3}$ & -1.964 \\
\hline
\end{tabular}

TABLE I

ESTIMATED PARAMETERS.

In order to invert equation 6 , equation 7 can be obtained. The solution for equation 7 , corresponds to equation 8 , resulting in a function with its domain from 0 to $5.955 \mathrm{Rad} / \mathrm{s}$, that has as input a velocity and as output the servo control signal.

$$
\begin{gathered}
\left(\omega b_{2}-a_{2}\right) d^{2}+\left(\omega b_{1}-a_{1}\right) d+\omega b_{0}-a_{0}=0 \\
d=\frac{-b \pm \sqrt{b^{2}-4 a c}}{2 a}
\end{gathered}
$$




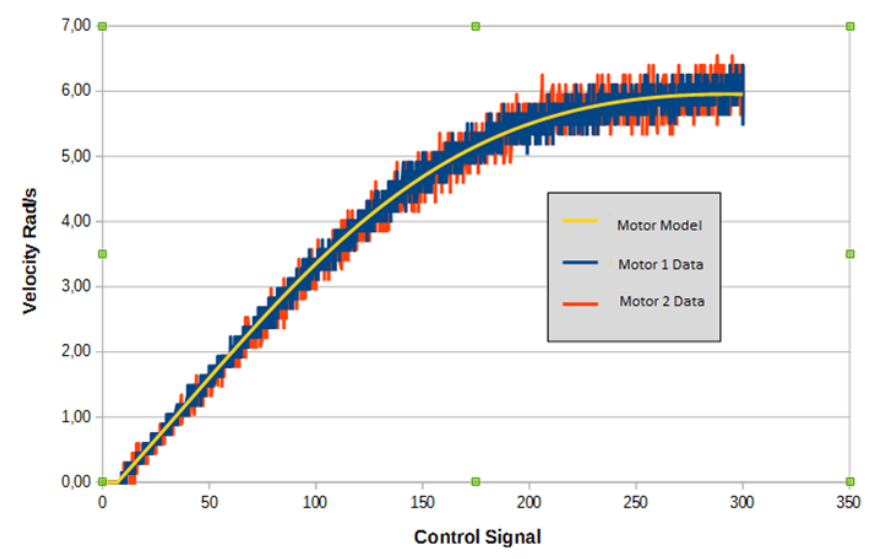

Fig. 8. Futaba S3003 Model.

where:

- $a=\omega b_{2}-a_{2}$

- $b=\omega b_{1}-a_{1}$

- $c=\omega b_{0}-a_{0}$

For an input inside the referred function's domain, equation 8 returns two values, the chosen value must be equal or greater than 7 and less or equal than 293. Values from 0 to 6 are inside the dead zone and values superior to 293 correspond to the saturation zone.

The function that describes the servo behavior in a larger domain is shown in Figure 9, where it can be seen that for a certain desired speed there can be two solutions, with one of the solutions always above the valid domain.

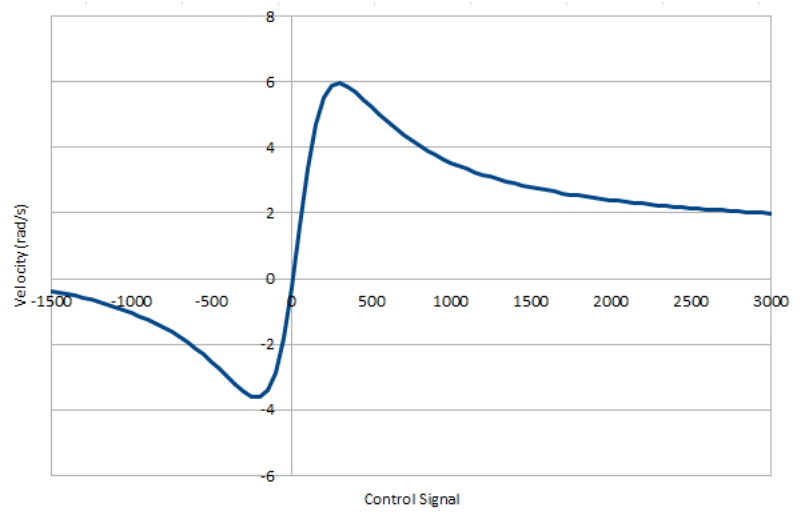

Fig. 9. Function in a larger domain

\section{EXTERnAL GLOBAL LOCALIZATION SySTEM}

An external vision based global localization system was developed, in order to provide, in real-time, the robot absolute localization, allowing the estimate of some robot parameters. A Raspberry Pi combined with its Camera Module, as shown in Figure 10, can act as a smart camera in the sense that some processing can be done on the Raspberry Pi and the results can be transmitted over the wired or the wireless network connection.

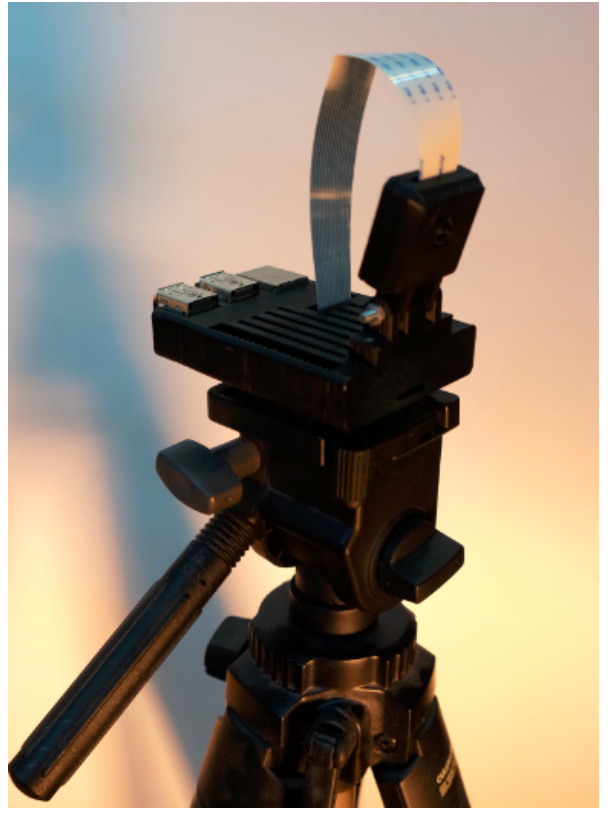

Fig. 10. Raspberry Pi combined with its Camera Module

As the computing power of a Raspberry Pi is limited, when compared to a standard PC, the amount of processing should be kept to a minimum. That can be achieved by using RGB LEDs as markers and very fast shutter delay. The result is a very dark image with only a few illuminated clusters where the LEDs are present, as shown in Figure 11.

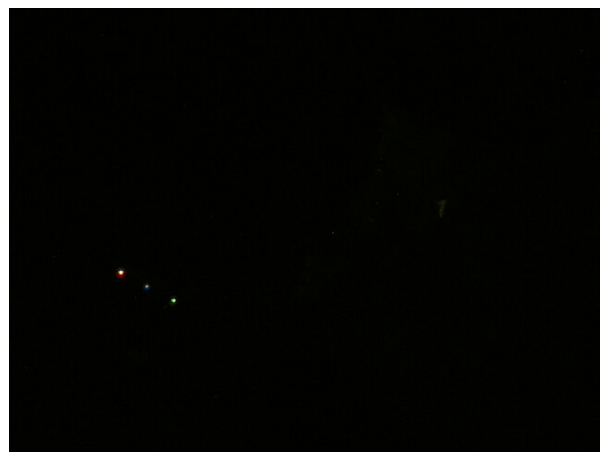

Fig. 11. Example Image

A combination of a simple threshold and a fast clustering algorithm can be used to isolate those clusters. Having the camera internal and external parameters and knowing the height of each RGB LED $z_{L}$, the $x$ and the $y$ position can be calculated. From the previously known relative position for the RGB LEDs relative to the robot center of rotation, the robot pose $\left(x_{R}, y_{R}, \theta_{R}\right)$ can be estimated. This pose can be sent from the Raspberry Pi to a computer application, which is also connected to the robot and can collect all relevant data, such as robot pose, wheel speed reference and line sensor values. 


\section{A. Robot Parameter Estimation}

From equations 1 and 2 it is straightforward to find that if the same speed $\left(\omega_{s}\right)$ is applied to each wheel, the robot will travel, as shown in Figure 12, in a straight line with $v=\omega_{s} . r$ and $\omega=0$. Logging this movement, from two poses $\left(x_{R}\left(k_{1}\right)\right.$, $\left.y_{R}\left(k_{1}\right), \theta_{R}\left(k_{1}\right)\right)$ and $\left(x_{R}(k 2), y_{R}\left(k_{2}\right), \theta_{R}\left(k_{2}\right)\right), \theta_{R}\left(k_{1}\right)$ and $\theta_{R}\left(k_{1}\right)$ should be roughly equal and the distance traveled, calculated by equation 9 , can be used to estimate the mean speed, applying equation 10, where $T$ is the sampling time. If the constant $r$ is known (from a measuring the wheel diameter) $w_{s}$ can be calibrated. In the case that $w_{s}$ is already calibrated this measure can be used to refine the value of $r$ as it can differ slightly from the measured value due to the softness of the tyre or also the floor material.

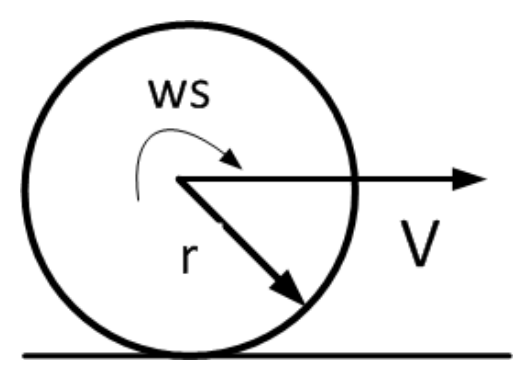

Fig. 12. Robot Wheel

$$
\begin{gathered}
d=\sqrt{(x R(k 2)-x R(k 1))^{2}+(y R(k 2)-y R(k 1))^{2}} \\
v m=\frac{d}{\left(k_{2}-k_{1}\right) * T}
\end{gathered}
$$

Another important parameter is $b$, the wheel distance. While this parameter can be directly measured it can be also estimated from a simple experiment. Logging a trajectory where one of the wheels is kept stopped and the other with a constant speed $\omega_{\omega}$. Again from equations 1 and 2 the robot angular velocity is given by equation 11 and, again, from two poses $\left(x_{R}\left(k_{1}\right), y_{R}\left(k_{1}\right), \theta_{R}\left(k_{1}\right)\right)$ and $\left(x_{R}\left(k_{2}\right), y_{R}\left(k_{2}\right)\right.$, $\left.\theta_{R}\left(k_{2}\right)\right)$, the mean angular speed can be given by equation 12 and can be used to estimate $b$, applying equation 13 .

$$
\begin{gathered}
\omega=\frac{\omega_{\omega} r}{b} \\
\omega_{m}=\frac{\theta_{R}\left(k_{1}\right)-\theta_{R}\left(k_{1}\right)}{\left(k_{2}-k_{1}\right) * T} \\
b=\frac{\omega_{\omega} \cdot r}{w m}
\end{gathered}
$$

\section{Mobile Robotics EXPERIMENT}

Following a line with a robot based on the differential kinematics is a classical introductory experiment that allows students to be introduced to the challenges of mobile robotics. Understanding the concepts of sensor, actuator and locomotion are the primary goals of this experience based on the control of a reactive robot [2]. Initially students attend to a lecture which has as contents the prototype description, the differential kinematics, and how to develop a controller for this specif challenge. The prototype description and its kinematics were described in the section II. In the next subsections a proposal for a controller, the educational experiment with hardware and the Student's Feedback are presented.

\section{A. Controller Proposal}

The goal of the robot is to follow a line as fast as possible, for that purpose it is necessary to maintain the highest possible linear velocity, with an angular velocity that is proportional to the path curvature. With the reflectance sensor, also known as line sensor, it is possible to know at each sample time the robot posture in relation to the line. By this way the path curvature is know, being proportional to the line distance to the sensor center (error).

The robot linear velocity can be given by the following equation:

$$
v=K_{1}-K_{3} \cdot \mid \text { error } \mid
$$

where $K_{1}$ indicates the top linear velocity and $K_{3}$ controls the velocity decrease, as a function of the path curvature.

The robot angular velocity can be given by the following equation:

$$
\omega=K_{2} . \text { error }
$$

where $K_{2}$ controls the angular velocity, as a function of the curvature path.

The robot's movement is controlled by varying each wheel velocity independently, by this way the velocity for each wheel can be given by equations 16 and 17, having in mind equations 1 and 2 .

$$
\begin{aligned}
& V_{1}=v+\frac{\omega b}{2} \\
& V_{2}=v-\frac{\omega b}{2}
\end{aligned}
$$

From equations 14, 15 and 16, equation 18 can be obtained:

$$
V_{1}=K_{1}+\frac{K_{2} \cdot \text { error.b }}{2}-K_{3} \cdot \mid \text { error } \mid
$$

From equations 14, 15 and 17, equation 19 can be obtained:

$$
V_{2}=K_{1}-\frac{K_{2} \cdot \text { error } . b}{2}-K_{3} \cdot \mid \text { error } \mid
$$




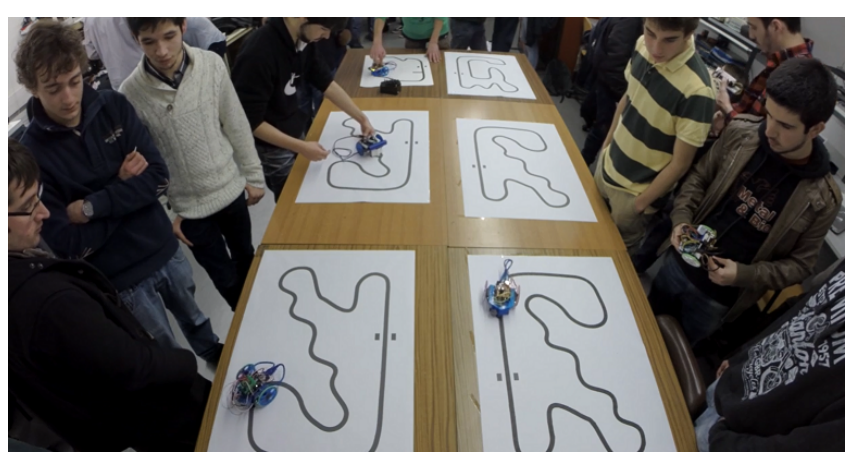

Fig. 13. Students at the Workshop

\section{B. Experiment With Hardware}

After the theoretical introduction, where students were introduced to the differential kinematics, the prototype hardware and the proposal of a controller for line following, they performed the robotics experiment with hardware. The students can be seen in Figure 13, at the workshop, working with robot prototypes.

The chosen educational robot challenge was a classical introductory experiment, that consisted in following a line with a mobile robot based on the differential kinematics, with the controller described in subsection IV-A. Students observed the effect on the robot's performance, while following a line, for different controller parameters. The work with hardware had the goal for the students to gain more sensitivity for the controller parameters choice, receiving an instantaneous feedback and having also the chance to compare and discuss their results with the ones obtained by their colleagues.

\section{Student's Feedback}

In order to receive feedback regarding the effectiveness of the mobile robotics experiment an inquire was made to the students that participated in the workshop. The inquire was performed by 24 Students, having for each question the option to answer from 1 to 5 , where 1 is "I totally disagree", 3 is "I am neutral" and 5 is "I totally agree". Bellow the inquire results can be found, where the average results and the standard deviation (STDV) of the students answers are presented.

\begin{tabular}{|l|c|c|}
\hline Question: & Average & STDV \\
\hline $\begin{array}{l}\text { The theoretical introduction was im- } \\
\text { portant in the learning process? }\end{array}$ & 3.9 & 1.3 \\
\hline $\begin{array}{l}\text { Practical laboratorial work was im- } \\
\text { portant in the learning process? }\end{array}$ & 4.5 & 0.8 \\
\hline $\begin{array}{l}\text { The work with a real robot was use- } \\
\text { ful for the workshop contents learn- } \\
\text { ing process? }\end{array}$ & 4.6 & 0.5 \\
\hline $\begin{array}{l}\text { Would you like to work again with } \\
\text { robots? }\end{array}$ & 4.5 & 0.7 \\
\hline $\begin{array}{l}\text { Working with robots is important to } \\
\text { motivate yourself? }\end{array}$ & 4.0 & 1.1 \\
\hline $\begin{array}{l}\text { Working with robots was useful to } \\
\text { understand control theory topics? }\end{array}$ & 4.0 & 0.9 \\
\hline
\end{tabular}

\section{CONClusions ANd Future Work}

In this paper it was presented an educational mobile robotics experiment based on a low cost mobile robot prototype. The mobile robot consists in a 3D printed small prototype, that uses inexpensive hardware, such as servo motors, an Arduino Uno platform and an infra-red detector array.

The prototyped robot has the possibility to communicate with external devices, allowing remote control and realtime monitoring. An external vision based global localization system was developed, in order to provide, in real-time, the robot absolute localization, allowing the estimate of some robot parameters.

The modeling information of a modified Futaba S3003 Servo-Motor, presented in this paper, is a relevant information, mainly for practitioners that use this servo in robot competitions, allowing them to learn how to modify it and to understand in detail the servo internal controller.

It was concluded from the inquire results that students were substantially motivated to participate in the workshop, mainly because the laboratory work involved mobile robots.

As future work the authors will organize other workshops with different robotic subjects.

\section{ACKNOWLEDGMENT}

This work is financed by the ERDF European Regional Development Fund through the Operational Programme for Competitiveness and Internationalisation - COMPETE 2020 Programme within project POCI-01-0145-FEDER-006961, and by National Funds through the FCT Fundação para a Ciência e a Tecnologia (Portuguese Foundation for Science and Technology) as part of project UID/EEA/50014/2013.

\section{REFERENCES}

[1] Borenstein, J., Everett, H., Feng, J. 'Where am I?' Sensors and Methods for Mobile Robot Positioning Technical Report, The University of Michigan 1996

[2] Pakdaman, M. Design and Implementation of Line Follower Robot Second International Conference on Computer and Electrical Engineering 2009

[3] Gregory Dudek and Michael Jenkin Computational Principles of Mobile Robotics Cambridge University Press 2000

[4] H. Choset, K. Lynch, S. Hutchinson, G. Kantor, W. Burgard, L. Kavraki, S. Thrun, Principles of Robot Motion : Theory, Algorithms, and Implementations, MIT Press, 2005.

[5] Lund, H., Pagliarinis, L. Robocup jr. with lego mindstorms in Proceedings of the 2000 IEEE International Conference on Robotics and Automation, San Francisco, CA, IEEE 2000

[6] A. Eguchi RoboCupJunior for promoting STEM education, 21st century skills, and technological advancement through robotics competition, Robotics and Autonomous Systems, 2016.

[7] Sklar, E., Eguchi, A., Johnson, J.,RoboCupJunior: Learning with Educational Robotics, AI Magazine, Volume 24, Number 2, 2003

[8] Lai. Toh1, A, Causo1, P. Tzuo, I, Chen, and S, YeoA Review on the Use of Robots in Education and Young Children, Educational Technology and Society, 2015

[9] Pololu http://www.pololu.com/product/1419 2017

[10] Montana State University, 2014 Futaba S3003 modification for continuous running, http://www.coe.montana.edu/ee/ecerover/servomod.html 2004

[11] Neves, D., Silva, M., Gonçalves J., Costa. Prototyping Small Robots for Junior Competitions: MicroFactory Case study 11th IFAC Symposium on Advances in Control Education 2016 